\title{
Coleópteros coprófagos (Scarabaeidae: Scarabeinae) de la Reserva Nacional Tambopata, Madre de Dios, Perú
}

\author{
Dung beetles (Scarabaeidae: Scarabeinae) from the Reserva Nacional \\ Tambopata, Madre de Dios, Peru
}

\author{
Luis Figueroa y Mabel Alvarado
}

Departamento de Entomología Museo de Historia Natural, Universidad Nacional Mayor de San Marcos, Av. Arenales 1256, AparMarcos, Av. Arenales 1256, Email Luis Figueroa: luis thecell@hotmail.com

\section{Resumen}

En este estudio se informa de las especies de Scarabaeinae (Coleoptera: Scarabaeidae) colectadas en la Reserva Nacional Tambopata, en el año 2009. Fueron colectados un total de 38 especies y 874 individuos. La tribu Canthonini tuvo la mayor cantidad de especies y abundancia. Se evidencia la preferencia de los hábitos coprófagos sobre los necrófagos en los escarabajos peloteros. Se comparan las especies colectadas en época lluviosa y de estío.

Palabras clave: Scarabaeinae, escarabajo del estiércol, Tambopata, Explorer's Inn, coprófagos.

\section{Abstract}

This study reports the species of Scarabaeinae (Coleoptera: Scarabaeidae) collected in Tambopata National Reserve in 2009. A total of 38 species and 874 individuals were collected. The tribe Canthonini showed the highest diversity and abundance. Coprophagy is clearly preferred over necrophagy by the dung beetle fauna in the area. A comparison of the species collected during the rainy and dry seasons is presented.

Keywords: Scarabaeinae, dung beetle, Tambopata, Explorer's Inn, coprófagos.

\section{Introducción}

Scarabaeinae es una subfamilia de escarabajos/coleópteros ampliamente distribuida y uno de los grupos más peculiares dentro de la superfamilia Scarabaeoidea (Hanski \& Camberfort 1991). Este es un grupo ecológicamente importante debido a que controla las poblaciones de moscas, fertiliza el suelo, recicla nutrientes y es un dispersor secundario de semillas (Ramirez 2009). Para el mundo, se reconocen aproximadamente 6000 especies, de estas, 1250 se registran para el Neotrópico (Escobar 2000).

Los Scarabaeinae forman un gremio muy bien definido tanto funcional como taxonómicamente, siendo un grupo claramente monofilético y bien representado en las áreas tropicales (Durães et al. 2005). La simplicidad y poco costo del sistema de muestreo de los Scarabaeinae los convierte en un grupo ideal para estudios comparativos entre distintas localidades de un mismo tipo de ecosistema (Escobar \& Chacón 2000). Los Scarabaeinae son un grupo excepcional para comparar paisajes donde originalmente han dominado los bosques, se puede comparar bosques con distinto grado de perturbación antropogénica, incluso aquellos donde la ganadería ha provocado una modificación muy importante (Favila \& Halffter 1999).

Diferentes estudios sobre uso y fragmentación de hábitats han mostrado una tendencia de cambio y disminución en la composición y abundancia de escarabajos coprófagos del hábitat natural al degradado (Lopera 1996). Estos trabajos también han aportado información sobre especies típicas del interior del bosque y especies de áreas abiertas. Otros estudios han contemplado evaluaciones de diversidad de escarabajos coprófagos en zonas de cultivos, transectos altitudinales y efecto de borde (Camacho 1999).

En el Perú, no se tiene registro de cuantas especies de Scarabaeidae existen, ni sobre su distribución, pero se pueden mencionar trabajos como los de Grados et al. (2010) que registraron 68 especies Scarabaeinae en los alrededores de Puerto Maldonado y Larsen et al. (2006) que estudiaron casos extremos de especialización en especies Scarabaeinae que ocupan nichos ecológicos inusualmente estrechos en Madre de Dios.

El presente trabajo informa de especies de Scarabaeidae colectados en la Reserva Nacional Tambopata.

\section{Material y métodos}

Para este estudio se realizaron dos muestreos, uno que corresponde a la época de lluvias (noviembre 2009) y otra a la época seca (mayo 2009). Los muestreos se realizaron en el Albergue Explorer's Inn, a orillas del Río Tambopata y el Río La Torre (12 $2^{\circ} 50.183^{\prime} \mathrm{S}, 69^{\circ} 17.627^{\prime} \mathrm{W} ; 161 \mathrm{~m}$ de altitud) dentro de la Reserva Nacional Tambopata en el Departamento de Madre de Dios. Este albergue está localizado en un bosque húmedo tropical (Curatola 2009).

Para la colecta se instalaron 30 trampas de caída con cebo, 15 con heces humanas (coprotrampas) y 15 trampas con carne en estado de descomposición (necrotrampas), las que estuvieron activas 48 horas. Las trampas se colocaron a lo largo de un transecto, con separación de $20 \mathrm{~m}$ entre trampa y trampa, debido a la longitud de la trocha, menor a $1 \mathrm{~km}$. Las trampas de caída son envases plásticos de 1 litro de capacidad de boca ancha, enterradas al ras del piso, con el cebo contenido en gasa y suspendido encima del frasco. El envase plástico contiene aproximadamente $300 \mathrm{~mL}$ de agua con jabón.

Las individuos colectados se colocaron en bolsas plásticas conteniendo alcohol de $96^{\circ}$, con su respectiva etiqueta, las que fueron trasladadas y depositadas en el Departamento de Entomología del Museo de Historia Natural de la Universidad Nacional Mayor de San Marcos (MUSM), donde se procedió 

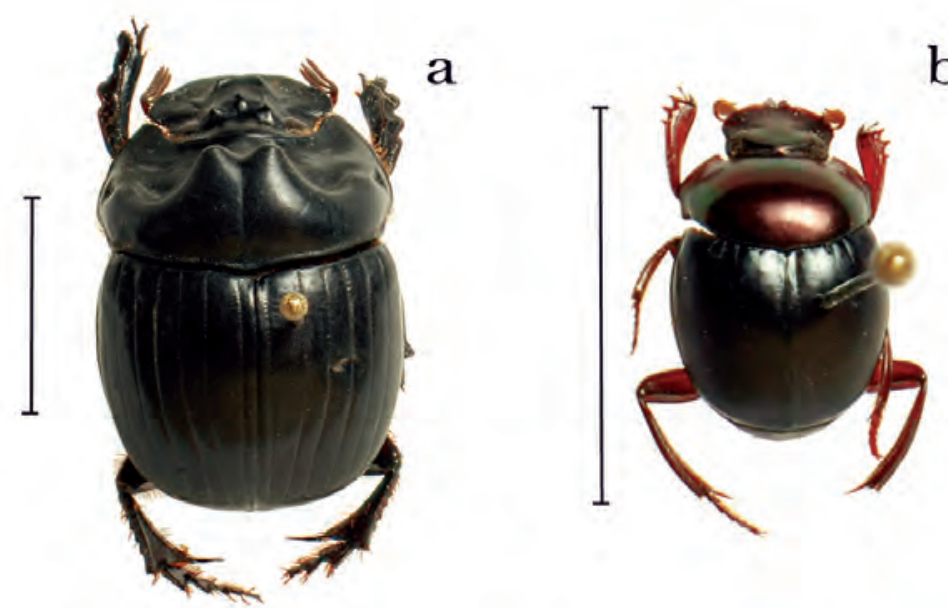

b
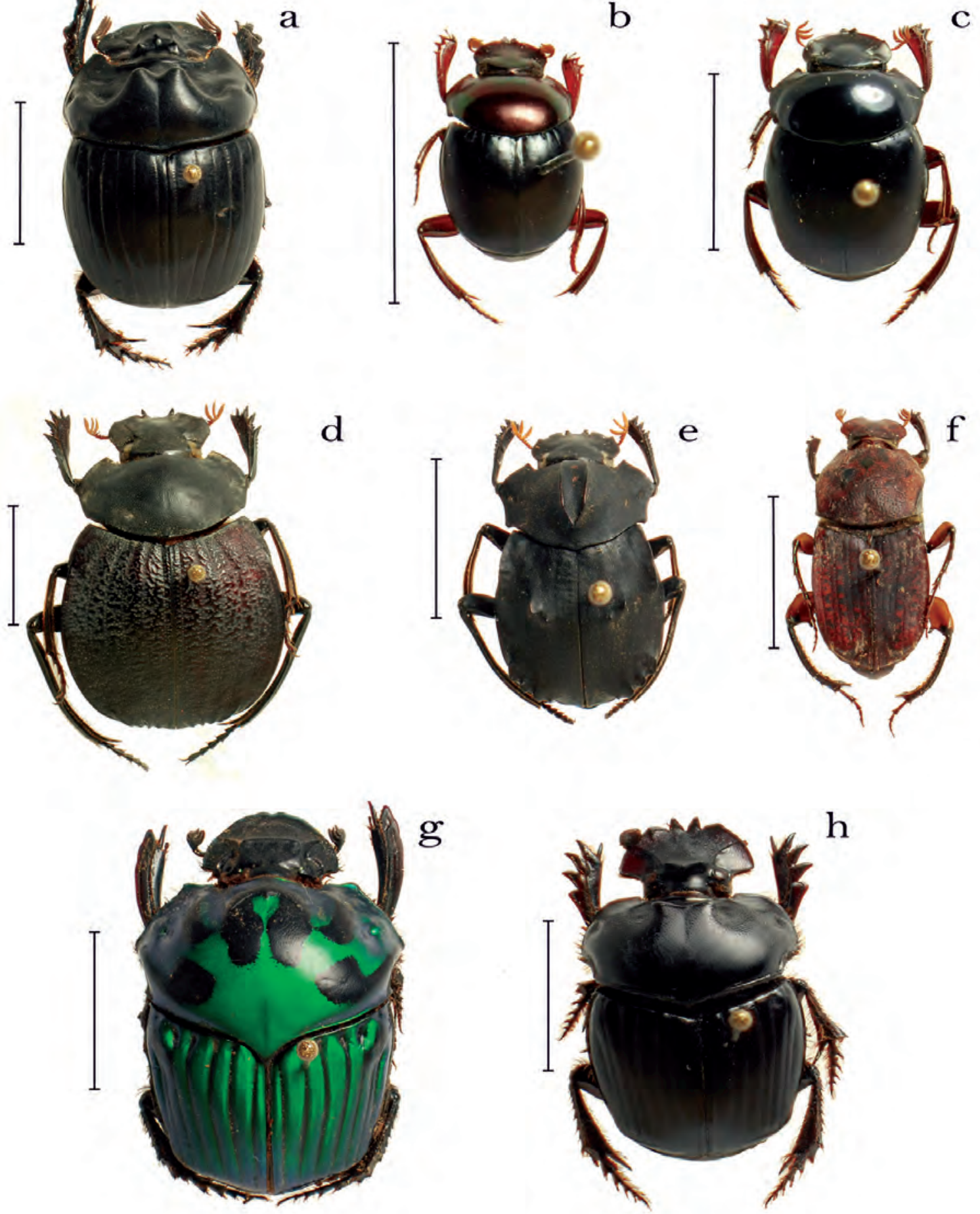

Figura 1. Vista dordal de algunas especies de Scarabaeidae capturados en la Reserva Nacional Tambopata en el año 2009. (a) Dichotomius prietoi, (b) Sylvicanthon bridarolli, (c) Canthon aequinotialis, (d) Deltochilum orbiculare, (e) Deltochilum carinatum, (f) Eurysternus caribaeus, (g) Oxysternon conspicillatum, (h) Coprophanaeus telamon. Barras indican $1 \mathrm{~cm}$.

a separar los escarabajos para su montaje, etiquetado e identificación con la ayuda de claves y comparación con la colección del MUSM.

\section{Resultados}

Se colectó un total de 874 individuos, 569 para la época de lluvias (noviembre) y 315 para la época de estío (mayo), registrándose 38 especies de la subfamilia Scarabaeinae (Coleoptera: Scarabaeidae) (Tabla 1, Fig. 1). En ambas épocas se encontró mayor abundancia en las coprotrampas, totalizando
632 especímenes y 35 especies (Tabla 2). En contraste, en las necrotrampas se recolectaron 242 individuos y 20 especies.

Se colectaron especies correspondientes a 6 de las 7 tribus presentes en el Neotrópico: Ateuchini, Canthonini, Coprini, Eurysternini, Phanaeini, Onthophagini. La tribu Sisyphini no tiene ocurrencia en Perú, ya que está distribuido principalmente en la región Etiópica y algunas especies habitan en Centro América.

Onthophagussp. (Grupo Clypeatus) fue la especie con mayor abundancia (100 individuos), esta especie tiene preferencia por 
Tabla 1. Especies de Scarabaeinae capturados en la Reserva Nacional Tambopata en el año 2009. Se indica la época de captura y el tipo de trampa.

\begin{tabular}{|c|c|c|c|c|c|}
\hline \multirow{3}{*}{ Tribu } & \multirow{3}{*}{ Especies } & \multicolumn{4}{|c|}{ Época } \\
\hline & & \multicolumn{2}{|c|}{ seca (mayo) } & \multicolumn{2}{|c|}{ lluvias (noviembre) } \\
\hline & & Necro & Copro & Necro & Copro \\
\hline ATEUCHINI & 1. Ateuchus sp & 2 & & & \\
\hline ATEUCHINI & 2. Ateuchus sp 2 & & 1 & & \\
\hline ATEUCHINI & 3. Ateuchus sp 3 & & & 3 & \\
\hline ATEUCHINI & 4. Canthidium nr. copricollis & 1 & 1 & & 4 \\
\hline ATEUCHINI & 5. Canthidium sp & & 1 & 3 & \\
\hline ATEUCHINI & 6. Uroxys sp & 1 & & 1 & \\
\hline CANTHONINI & 7. Anisocanthon villosus (Harold, 1868) & & & & 9 \\
\hline CANTHONINI & 8. Canthon aequinotialis Harold, 1868 & & 12 & & \\
\hline CANTHONINI & 9. Canthon smaragdulus (Fabricius, 1781) & & 2 & & \\
\hline CANTHONINI & 10. Canthon luteicollis Erichson, 1847 & 1 & 3 & & 4 \\
\hline CANTHONINI & 11. Canthon monilifer Blanchard 1846 & & 2 & & 89 \\
\hline CANTHONINI & 12. Canthon quinquemaculatus Castelnau, 1840 & 12 & 5 & & \\
\hline CANTHONINI & 13. Canthon $\mathrm{sp} 1$ & & 2 & & 1 \\
\hline CANTHONINI & 14. Deltochilum amazonicus Bates, 1887 & 3 & 25 & & \\
\hline CANTHONINI & 15. Deltochilum carinatum (Westwood, 1837) & 9 & & & 3 \\
\hline CANTHONINI & 16. Deltochilum orbiculare Lansberge, 1874 & 14 & 11 & & \\
\hline CANTHONINI & 17. Deltochilum sp & 26 & 2 & 2 & \\
\hline CANTHONINI & 18. Scybalocanthon $\mathrm{sp}$ & 3 & 1 & 5 & 32 \\
\hline CANTHONINI & 19. Sylvicanthon bridarolli Martinez, 1949 & & 2 & & 5 \\
\hline COPRINI & 20. Dichotomius conicollis (Blanchard, 1846) & & & & 1 \\
\hline COPRINI & 21. Dichotomius mamillatus (Felsche,1901) & & 1 & & \\
\hline COPRINI & 22. Dichotomius nr. batesi & 67 & 3 & 6 & 6 \\
\hline COPRINI & 23. Dichotomius prietoi Martinez \& Martinez, 1982 & & 87 & & 2 \\
\hline COPRINI & 24. Dichotomius robustus (Luederwaldt, 1935) & & & & 3 \\
\hline COPRINI & 25. Dichotomius worontzowi (Pereira, 1942) & & 1 & & \\
\hline EURYSTERNINI & 26. Eurysternus caribaeus Herbst 1789 & 12 & 16 & & 25 \\
\hline EURYSTERNINI & 27. Eurysternus hamaticollis Balthasar 1939 & & 1 & & \\
\hline EURYSTERNINI & 28. Eurysternus hypocrita Balthasar 1939 & 3 & 1 & & \\
\hline EURYSTERNINI & 29. Eurysternus sp. & & 1 & & \\
\hline ONTHOPHAGINI & 30. Onthophagus sp. (group clypeatus) & & & 16 & 84 \\
\hline ONTHOPHAGINI & 31. Onthophagus haematopus Harold, 1875 & & 46 & & 2 \\
\hline ONTHOPHAGINI & 32. Onthophagus sp.1 & 12 & 28 & & \\
\hline ONTHOPHAGINI & 33. Onthophagus sp.2 & 1 & 66 & 2 & \\
\hline ONTHOPHAGINI & 34. Onthophagus sp.3 & & 8 & & \\
\hline PHANAEINI & 35. Coprophanaeus sp. & 17 & 2 & 4 & \\
\hline PHANAEINI & 36. Coprophanaeus telamon Erichson 1847 & 14 & 1 & 2 & \\
\hline PHANAEINI & 37. Oxysternon conspicillatum Weber, 1801 & & 26 & & \\
\hline PHANAEINI & 38. Phanaeus chalcomelas Perty. 1830 & & 3 & & 1 \\
\hline Abundancia & & 198 & 361 & 44 & 271 \\
\hline Riqueza & & 17 & 29 & 10 & 15 \\
\hline
\end{tabular}

Tabla 2. Comparación entre la Riqueza de especies y Abundancia por tribus de Scarabaeinae capturados en la Reserva Nacional Tambopata en el año 2009. Necro= necrotrampas, Copro= coprotrampas.

\begin{tabular}{|c|c|c|c|c|c|c|c|c|}
\hline \multirow[t]{3}{*}{ Tribus } & \multicolumn{4}{|c|}{ Riqueza } & \multicolumn{4}{|c|}{ Abundancia } \\
\hline & \multicolumn{2}{|c|}{ seca (mayo) } & \multicolumn{2}{|c|}{ lluvias (noviembre) } & \multicolumn{2}{|c|}{ seca (mayo) } & \multicolumn{2}{|c|}{ lluvias (noviembre) } \\
\hline & Necro & Copro & Necro & Copro & Necro & Copro & Necro & Copro \\
\hline Ateuchini & 3 & 3 & 3 & 1 & 4 & 3 & 7 & 4 \\
\hline Canthonini & 7 & 11 & 2 & 7 & 68 & 67 & 7 & 143 \\
\hline Coprini & 1 & 4 & 1 & 4 & 67 & 92 & 6 & 12 \\
\hline Eurysternini & 2 & 4 & 0 & 1 & 15 & 19 & 0 & 25 \\
\hline Onthophagini & 2 & 4 & 2 & 2 & 13 & 148 & 18 & 86 \\
\hline Phanaeini & 2 & 4 & 2 & 1 & 31 & 32 & 6 & 1 \\
\hline
\end{tabular}




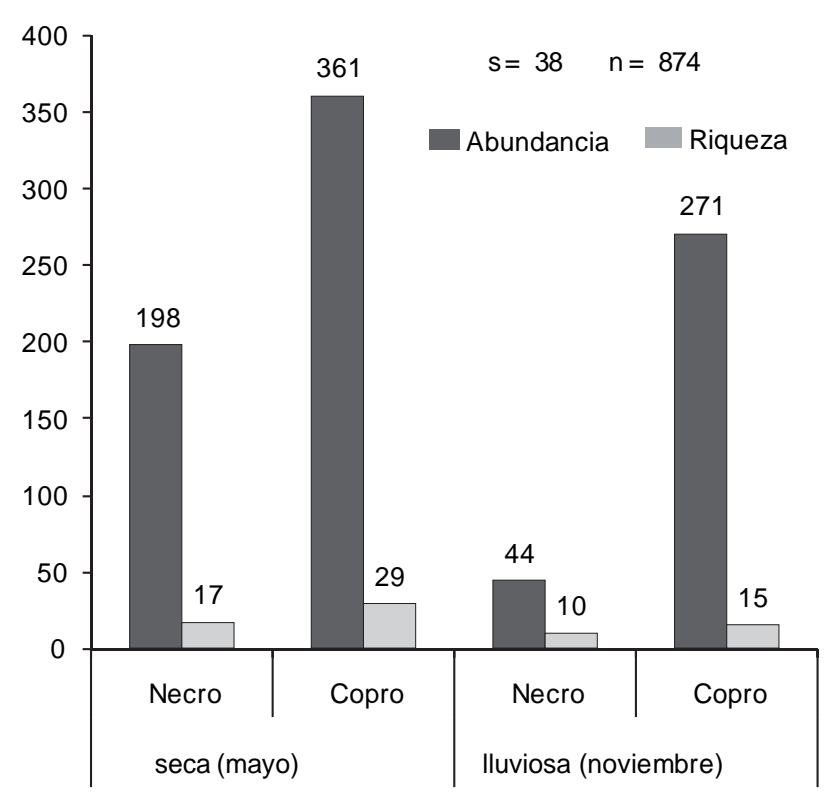

Figura 2. Abundancia y Riqueza especifica de Scarabaeidae capturados en la Reserva Nacional Tambopata en el año 2009; por tipo de cebo y por época de lluvias (noviembre) y seca (mayo).

los cebos de heces humanas (84\%). En el caso de la coprotrampas, Canthon monilifer Blanchard presenta mayor abundancia en este tipo de trampa con 91 especímenes, representando el 14,4\%. Para las necrotrampas, Dichotomius nr. batesi fue la más abundante con 73 especímenes siendo el 30,2\%.

Al comparar los muestreos, resalta la mayor riqueza específica y abundancia del muestreo en la época lluviosa (Fig. 2). Canthonini fue la tribu que registró la mayor riqueza específica, con 13 especies, lo que evidencia la preferencia de la tribu a la coprofagia, esto también es evidente en la Coprini.

\section{Discusión y conclusiones}

Las trampas pitfall con cebo de heces humanas son mejores atrayentes para los Scarabaeinae, dando como resultado, mayor riqueza específica y abundancia.

La tribu Canthonini al ser principalmente especies "rodadoras" (transportan las heces algunos metros) fueron atraídos principalmente por las heces humanas. Es significativa también la presencia de la tribu Onthophagini, la mayoría de especies de esta tribu son "tuneleros", es decir, entierran sus bolas de heces o carroña en el mismo lugar. El género Coprophanaeus tiene una mayor afinidad por las necrotrampas, esto debido al comportamiento carroñero.

La riqueza especifica del lugar, comparado con los resultados de Grados et al. (2010), es menor en 42\%, posiblemente debido a que este muestreo fue ejecutado en un solo tipo de formación vegetal, bosque húmedo tropical.

La época seca presentó menor riqueza y abundancia en estos muestreos. Esto debido a la ocurrencia de un fuerte "friaje" (temperaturas de $13^{\circ} \mathrm{C}$ ) que azotó la zona los días de la evaluación.

Las especies colectadas tienen amplia distribución en el llano Amazónico peruano, desde Loreto hasta Cuzco y Madre de Dios, no se reportan especies raras ni endémicas.

\section{Agradecimientos}

Este trabajo se realizó gracias al financiamiento de TReesPerú. Al albergue Explorer's Inn, por la facilidades en el hospedaje, en especial a Max Gunther por el apoyo constante a la ciencia. Al INRENA, que nos brindó los permisos de colecta No 055 c/c-2008-INRENA-IANP. A Diana Silva y Juan Grados por sus comentarios y sugerencias.

\section{Literatura citada}

Camacho R.A. 1999. Usos de las cercas vivas por parte de los escarabajos coprófagos (Scarabaeidae: Scarabaeinae) en un ambiente fragmentado del piedemonte llanero, Meta, Colombia. Tesis de Grado Pontificia Universidad Javeriana, Santafé de Bogotá.

Curatola G. 2009. Patrones de distribución espacial de Triplaris americana en Tambopata, Perú. Tesis para obtener el título de licenciada en Geografía y Medio Ambiente de la Pontificia Universidad Catolica del Perú.

Durães R., W. Martins, \& F. Vaz de Mello. 2005. Dung Beetle (Coleoptera: Scarabaeidae) Assemblages across a Natural Forest-Cerrado Ecotone in Minas Gerais, Brazil. Neotropical Entomology 34(5):721-731

Escobar F. \& P. Chacón. 2000. Distribución espacial y temporal en un Gradiente de Sucesión de la fauna de Coleopteros Coprofagos (Scarabaeinae, Aphodiinae) en un bosque tropical montano, Nariño - Colombia. Revista de Biología Tropical 48 (4): 961- 975.

Escobar F. 2000. Diversidad y Distribución de los Escarabajos del Estiércol (Coleoptera: Scarabaeidae: Scarabaeinae) de Colombia. PRIBES: 197-210.

Favila M.E., \& G. Halffter. 1999. Los Scarabaeinae (Insecta: Coleoptera) en el Monitoreo de la Diversidad Biológica: Ensayos perspectivas, pp. 225-241. En S. D. Matteucci, O. T. Solbrig, J. Morello, \& G. Halffter, Biodiversidad y uso de la Tierra, Conceptos y ejemplos de Latinoamerica. EUDEBA-UNESCO, Buenos Aires.

Grados J., L. Figueroa \& M. Alvarado. 2010. Insectos: Scarabaeinae (Coleoptera) y Arctiidae (Lepidoptera). Pp. 103-120. En: Figueroa, J. \& M. Stucchi. Eds. Biodiversidad de los Alrededores de Puerto Maldonado, Linea Base Ambiental del EIA del Lote 111, Madre de Dios. IPyD Ingenieros y AICB. Lima, Perú. 224 pp.

Hanski I. \& Y. Camberfort. 1991. Princeton University Press, Princeton. Competition in dung beetles. In: Hanski, I. \& Y. Cambefort, Eds. Dung Beetle Ecology. 305-329.

Larsen T.H., A. Lopera, \& A. Forsyth. 2006. Extreme trophic and habitat specialization by Peruvian dung beetles (Coleoptera: Scarabaeinae). Coleopterists Bulletin 60: 315-324.

Lopera A. 1996. Distribución y diversidad de escarabajos coprófagos (Scarabaeidae: Coleoptera) en tres relictos de bosque altoandino (Cordillera Oriental, Vertiente Occidental, Colombia). Tesis, Biólogo. Facultad de Ciencias Biológicas, Pontificia Universidad Javeriana, Santafé de Bogotá.

Ramirez P. 2009. Altitudinal variation and diversity of dung beetle (Scarabaeidae: Scarabaeinae) assemblages in the Peruvian cloud forest. Tesis para obtener el grado de MSc. del Imperial College London. 